\title{
Bilateral facial paralysis as the sole presenting feature of sarcoidosis: Report of a case
}

\author{
WILLIAM E. McINTOSH, D.O. \\ JOHN F. BRENNER, D.O. \\ JOHN E. ASCHENBRENNER, Ph.D \\ Fort Worth, Texas
}

The following is a case of a 32-year-old patient who presented with bilateral facial paralysis as the sole manifestation of sarcoidosis. Her initial physical examination failed to reveal any other clinical evidence of sarcoidosis. Her chest $x$-ray and laboratory studies were normal, with the exception of a mild cerebrospinal fluid pleocytosis and mildly elevated protein. The patient initally improved on a short course of corticosteroid therapy only to relapse after corticosteroids were withheld. Within 5 weeks she had signs and symptoms, chest $x$-ray findings and laboratory abnormalities consistent with the diagnosis of sarcoidosis. The diagnosis was confirmed by parotid gland biopsy. The patient made substantial improvement on corticosteroid therapy.

Bilateral peripheral facial paralysis is a rare clinical condition. Various etiologies have been reported, including Bell's palsy, ${ }^{1-5}$ diabetes mellitus, ${ }^{6}$ infectious mononucleosis, ${ }^{7}$ Stevens-Johnson syndrome,$^{8}$ sarcoidosis, ${ }^{9-11}$ herpes zoster ophthalmicus, ${ }^{12}$ ethylene glycol ingestion, ${ }^{13}$ Guillain-Barré syndrome, ${ }^{14}$ congenital anomalies such as Möbius syndrome, central nervous system infection, neoplastic diseases, periarteritis nodosa, and Melkersson-Rosenthal syndrome. ${ }^{15}$ Facial diplegia can also be found in degenerative diseases, such as progressive supranuclear palsy and bulbar palsy, neuromuscular junction diseases (myasthenia gravis and Eaton-Lambert syndrome), and also in some of the muscular dystrophies. ${ }^{16}$

The following is a report of a case of a young woman presenting with bilateral facial paralysis as the only clinical manifestation of sarcoidosis. A review of the literature failed to reveal a single case in which bilateral facial paralysis was the only manifestation of the disease. Her clinical course is followed and a brief discussion of sarcoidosis is included.

\section{Report of case}

A well-developed, well-nourished 32-year-old black/oriental woman visited her family physician for outpatient evaluation of right facial paralysis, which she reported had been acute in onset and had been accompanied by decreased taste sensation on the right side of her tongue. The patient was diagnosed as having Bell's palsy, and a regimen of steroids was prescribed. However, when she returned to her family physician 7 days later, the facial paralysis and decreased sense of taste had become bilateral.

The patient was hospitalized for further evaluation and treatment, and neurologic consultation was obtained. Her review of systems was negative except for some pain behind the right ear, which had preceded the onset of right facial numbness. Her past medical history was unremarkable, and there was no recent history of head trauma.

On physical examination, the patient was afebrile and had normal vital signs. The head, eyes, ears, nose, and throat were all normal. The neck was supple, with a midline trachea; there was no thyromegaly, cervical mass, or adenopathy. No bruits were heard. Auscultation of the heart and lungs was nonremarkable. The abdomen was soft and nontender, without organomegaly or masses. Neurologically, the patient was normal, except for the complete bilateral peripheral facial nerve paralysis and decreased taste on the anterior portion of the tongue.

Radiographic studies revealed normal posteroanterior and lateral chest x-rays, skull series, computed tomographic scan of the brain, and tomographs of the internal auditory canals. The patient's admission electrocardiogram was normal.

The complete blood count revealed a leukocyte 
count of $10,400 / \mathrm{cu}$. mm., with a differential count of 82 percent neutrophils and 18 percent lymphocytes. The hemoglobin concentration and hematocrit reading were $13.8 \mathrm{gm} . / \mathrm{dl}$. and 43.5 percent, respectively. The erythrocyte sedimentation rate was $5 \mathrm{~mm} . / \mathrm{hr}$., and coagulation study results were normal. The SMA-12 battery results and serum electrolyte and calcium values were within normal limits. The VDRL test was nonreactive, and urinalysis findings were normal. Lumbar puncture was performed, with the following results: cerebrospinal fluid (CSF) protein, $58 \mathrm{mg} . / \mathrm{dl}$.; CSF glucose, $49 \mathrm{mg} . / \mathrm{dl}$.; and 57 red blood cells and 13 white blood cells. Acid-fast, Gram stain, and culture studies of the CSF were all negative, as was CSF VDRL testing. Serum mono spot testing and antinuclear antibody studies were negative. A thyroid series was unremarkable. The angiotensinconverting enzyme value was normal at $23 \mathrm{U} / \mathrm{L}$.

The patient was diagnosed by exclusion as having bilateral Bell's palsy, and she was begun on prednisone according to the regimen of Adour. ${ }^{2}$ This patient remained at type II status, according to Adour's ${ }^{2}$ classification; therefore, the medication was continued for a total of 21 days. Following discharge, she initially improved on the steroid therapy, but after discontinuation the facial paralysis worsened. In addition, she developed significant hearing loss in her right ear, difficulty in swallowing, low-grade fever, and right parotid gland enlargement.

Five weeks after her initial discharge, the patient was readmitted to the hospital; she was found to have bilateral peripheral facial nerve weakness, bilateral cranial nerve VII dysfunction, and mild right-sided palatal weakness. The admission chest $\mathrm{x}$-ray revealed right peritracheal and hilar adenopathy. The angiotensin-converting enzyme value had become elevated to 43 (upper limit of normal, 30 ). Right parotid gland biopsy demonstrated noncaseating granuloma (Figs. 1-4). All remaining studies, including purified protein derivative intermediate skin testing, were negative. Steroid treatment was reinstituted, and the patient was dismissed on the seventh hospital day on a regimen of $60 \mathrm{mg}$. of prednisone daily.

Two years later, the patient had been taken off of all corticosteroid therapy. Her chest x-ray had returned to normal, and there was no palpable lymph node or salivary gland enlargement. She had greater than 90 percent resolution of the facial nerve paralysis.

\section{Discussion}

The only abnormality other than facial paralysis on this patient's admitting work up was lymphocytic spinal fluid pleocytosis and a slight elevation

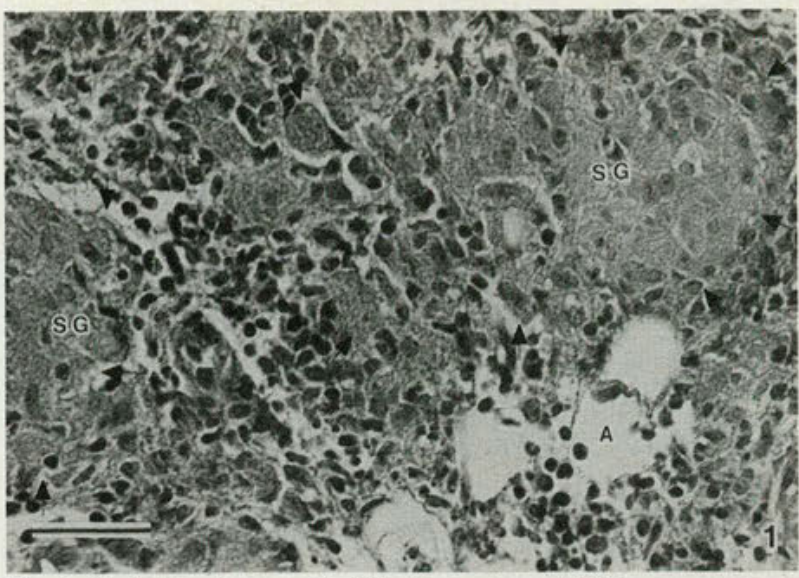

Fig. 1. A photomicrograph of the human parotid biopsy specimen showing moderate lymphocytic infiltration and sarcoid granuloma formation ( $S G$, arrows). The granulomas completely obliterate the parenchymal secretory units normally associated with this glandular tissue. Note the diffuse character of the sarcoid lesions and their epithelioid composition. Necrosis was not observed as an associated feature of these entities. Several adipose elements $(A)$ are also present in the lower right hand field of view. Bar represents $50 \mu \mathrm{m}$. Stained with Hematoxylin and Eosin ( $H$ \& E).

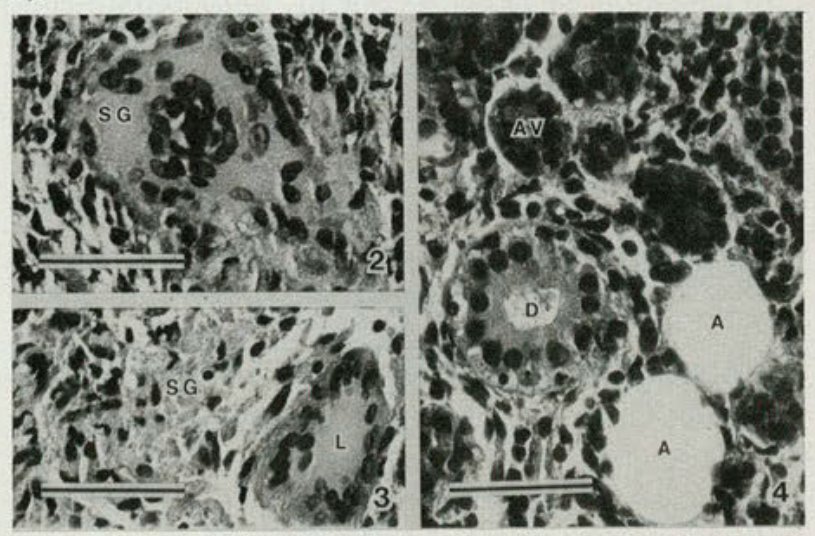

Fig. 2. The epithelioid cells comprising a single sarcoid granuloma (SG) are demonstrated. These cells were often arranged haphazardly, but in this structure there was a tendency for them to assume a circumferential pattern. Bar represents $50 \mu \mathrm{m} . \mathrm{H}$ \& $E$ stain. Fig. 3. A Langerhans giant cell $(L)$ occupies a position at the surface of a sarcoid granuloma (SG). Note the peripheral position of the nuclei in this cell type. Langerhans giant cells and foreign body giant cells have been described in association with sarcoid type lesions. Bar represents $50 \mu \mathrm{m}$. H \& E stain. Fig. 4. The majority of the inflammatory cells which are distributed between the alveolar units (AV) in this field of view are lymphocytes. Note the ductile element $(D)$ surrounded by these cells. Two adipose cells $(A)$ and some fibroblasts are also present within the stroma. Areas of the tissue not involved in granuloma formation were moderately to heavily involved in an inflammatory process. Bar represents $50 \mu \mathrm{m}$. $H$ \& E stain

in the CSF protein level. This CSF finding is common in patients with central nervous system or cranial neuropathies associated with sarcoidosis. It is a nonspecific finding, and, presumably, it reflects irritative or inflammatory lesions involving the central nervous system parenchyma, meninges, or cranial nerves near their origin. Follow up of the case clearly demonstrated sarcoidosis presenting 
as isolated, bilateral facial paralysis, with no other stigmata of the disease.

Sarcoidosis is a systemic disease, with multiple organ involvement; nervous system involvement occurs in 5 percent of cases. ${ }^{17}$ Microscopically, the fundamental sarcoid lesion consists of a focal collection of epithelioid cells with surrounding lymphocytes. Coalescence of macrophages leads to giant cell and epithelioid granulomas, which are noncaseating in type. ${ }^{18}$ The etiology of sarcoidosis remains unknown. While many hypotheses have been offered, none as yet satisfactorily explains this complicated disease phenomenon. Among the favorite current speculative causes are the following: an immunologic disorder characterized by lymphoproliferation and depressed, delayed-type hypersensitivity, increased T4 helper cells, overreactive B-cells, and circulating immune complexes; an infectious process; an atypical hypersensitivity reaction; and a genetic disorder. ${ }^{19}$

Pulmonary involvement is almost universal ( $>95$ percent of cases). ${ }^{20}$ Serum angiotensin-converting enzyme activity is increased in the majority of patients. ${ }^{11,21}$ Involvement of the facial nerve is a relatively common neurologic finding in sarcoidosis; however, bilateral involvement is very uncommon, and a neurologic symptom as the presenting complaint is also uncommon. ${ }^{11}$

Cecil's Textbook of Medicine ${ }^{22}$ refers to corticosteroid therapy as the mainstay of treatment of sarcoidosis around the world. There is no specific treatment for sarcoidosis and, in fact, most patients do not require any form of therapy. Corticosteroid medication is indicated particularly for patients with the following: ocular, myocardial, or lacrimal and salivary gland involvement; breathlessness; an abnormal chest $\mathrm{x}$-ray associated with a significant elevation of serum angiotensin-converting enzyme, positive gallium scan, or abnormal bronchoalveolar fluid; persistent hypercalcemia and/or hypercalciuria; disfiguring skin lesions; or central nervous system sarcoidosis. ${ }^{22}$ I would add one further indication-multiple cranial nerve involvement. Prednisone, $30 \mathrm{mg}$. daily, has been shown to provide adequate therapy. However, steroids have been shown to alter the long-term changes associated with sarcoidosis and relapses are common. ${ }^{23}$

\section{Summary}

A review of the literature revealed no other case report of sarcoidosis in which bilateral facial paralysis was the only presenting manifestation of the disease. Facial paralysis in sarcoidosis is an indication for steroid therapy, which can produce dramatic results. This case illustrates the difficulty in determining the etiology of bilateral facial paralysis, as well as the need for extensive evaluation and follow up.

1. Crumley, R.L.: Diagnosis and treatment of idiopathic facial paralysis. Primary Care 9:429-37, Jun 82

2. Adour, K.K.: Current concepts in neurology. Diagnosis and management of facial paralysis. N Engl J Med 307:348-51, Aug 82

3. Peitersen, E.: The natural history of Bell's palsy. Am J Otol 4:107-11, Oct 82

4. McGovern, F.H.: Bilateral Bell's palsy. Laryngoscope 75:1070-80, Jul 65

5. Goff, C.W.: Bilateral Bell's palsy. Am J Dis Child 137:83, Jan 83

6. Hattori, T., and Schlagenhauff, R.E.: Bilateral facial palsy occurrence with diabetes mellitus. NY State Med J 77:1492-4, Aug 77

7. Weintraub, M.I.: Bilateral facial palsy. A rare presentation of infectious mononucleosis. Clin Pediatr 16:1158-9, Dec 77

8. Friedman, J.H., Challenor, Y., and Brust, J.C.M.: Bilateral facial palsy associated with Stevens-Johnson syndrome. Neurology 29:1304-6, Sep 79

9. Wiederholt, W.C., and Siekert, R.G.: Neurological manifestations of sarcoidosis. Neurology 15:1147-54, Dec 65

10. James, E.G., and Sharma, O.P.: Neurological complications of sarcoidosis. Proc R Soc Med 60:1169-72, Nov 67

11. Delaney, P.: Neurologic manifestations in sarcoidosis. Review of the literature, with a report of 23 cases. Ann Intern Med 87:336-45, Sep 77

12. Shoji, H., et al.: A case of facial diplegia following herpes zoster ophthalmicus. Eur Neurol 19:327-9, 1980

13. Berger, J.R., and Ayyar, D.R.: Neurological complications of ethylene glycol intoxication. Report of a case. Arch Neurol 38:724-6, Nov 81

14. Gorman, J.B., Nagler, B., and Botton, J.E.: Facial diplegia and its causes. V Med 104:544-6, Aug 77

15. Rontal, E., and Sigel, M.E.: Bilateral facial paralysis. Laryngoscope 82:607-16, Apr 72

16. Adams, R.D., and Victor, M.: Principles of neurology. Ed. 3, McGrawHill Book Company, New York, 1985

17. Guenter, C.A., and Welch, M.H., eds.: Pulmonary medicine. Ed. 2, J.B. Lippincott Company, Philadelphia, 1982

18. Brown, J.K., and Ludmer, P.: New and old concepts in sarcoidosis. West J Med 138:546-51, Apr 83

19. James, D.G., and Williams, W.J.: Immunology of sarcoidosis. Am J Med 72:5-8, Jan 82

20. Selroos, O.: The diagnosis of sarcoidosis. Eur J Respir Dis 62:219-22, Aug 81

21. Rohatgi, P.K.: Significance of serum angiotensin converting enzyme and gallium scan in noninvasive diagnosis of sarcoidosis. Eur J Respir Dis 62:223-30, Aug 81

22. Wyngaarden, J.B., and Smith, L.H.: Cecil's Textbook of medicine. Ed. 16. W.B. Saunders Co., Philadelphia, 1982

23. Braunwald, E., et al., eds.: Harrison's Principles of internal medicine. Ed. 11, McGraw-Hill Book Company, New York, 1987, p. 1450.

Accepted for publication in February, 1986. Updating, as necessary, has been done by the authors.

Dr. McIntosh is an associate professor of medicine (neurology) at the Texas College of Osteopathic Medicine, Fort Worth, Texas. Dr. Brenner is in the private practice of internal medicine, and Dr. Aschenbrenner is in the Department of Anatomy at TCOM.

Dr. McIntosh, TCOM Medicine Clinic, 999 Montgomery, Fort Worth, Texas 76107. 


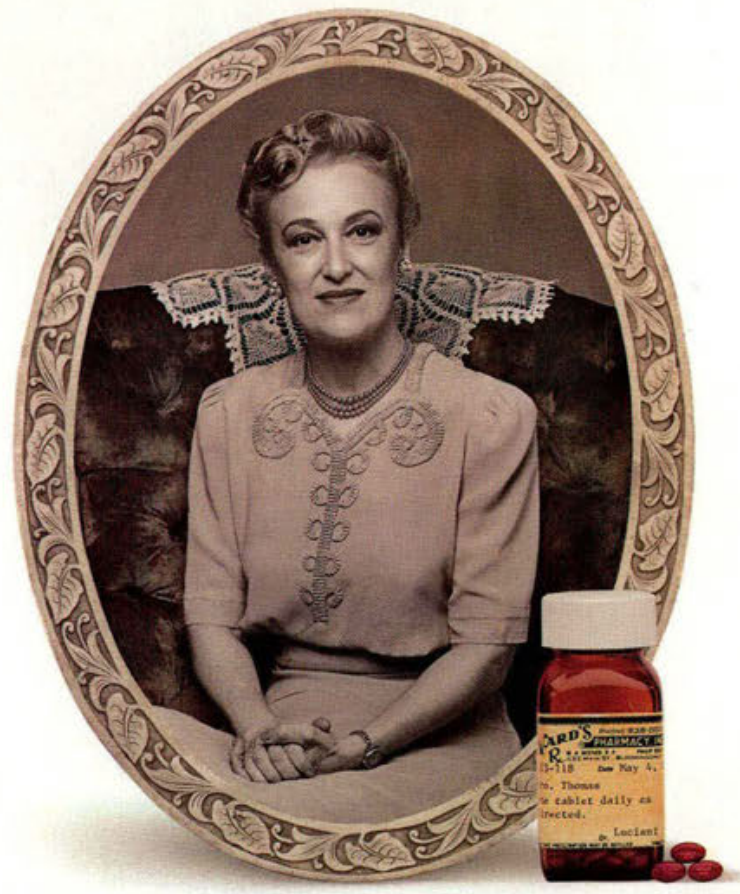

Now
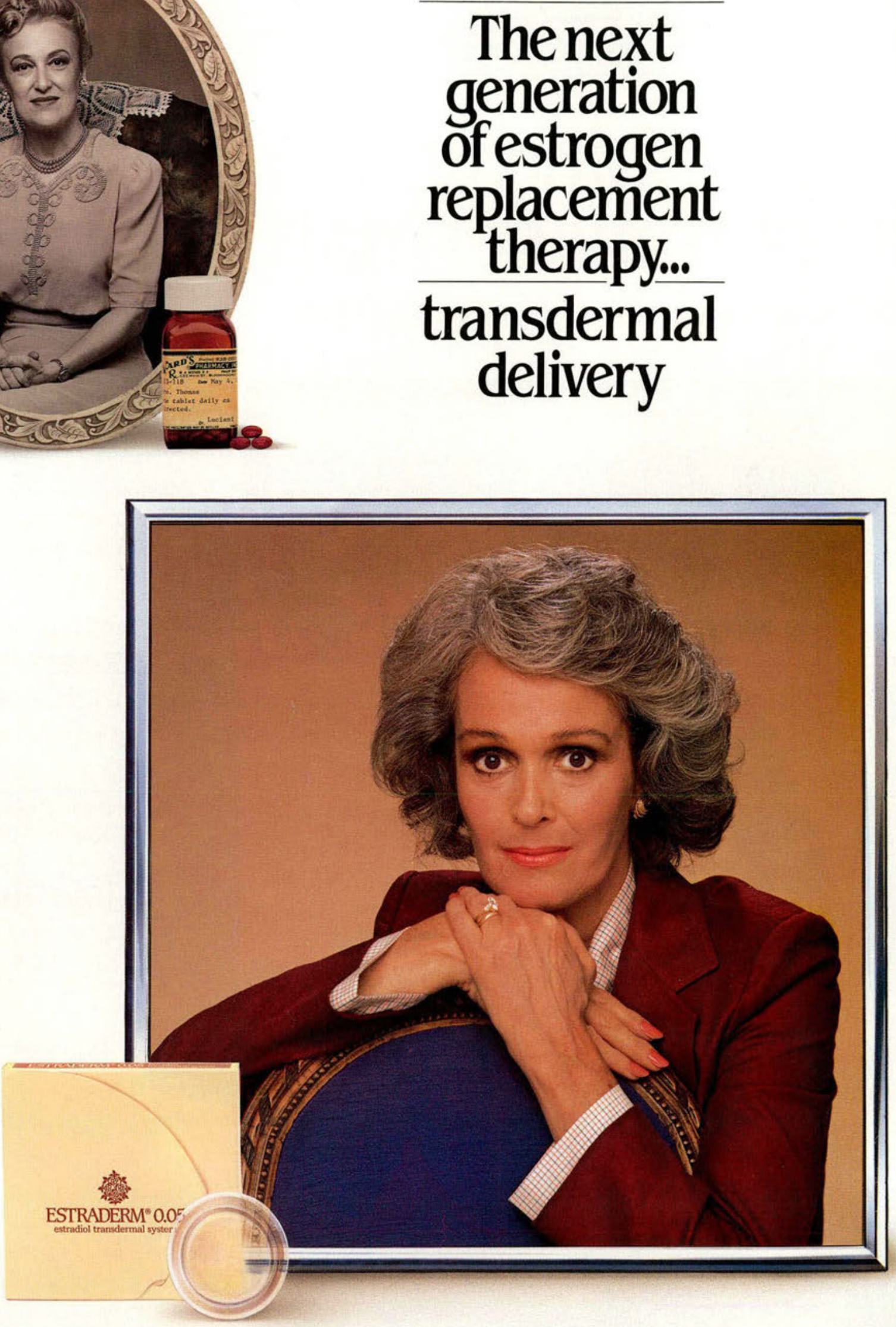


\section{NEW
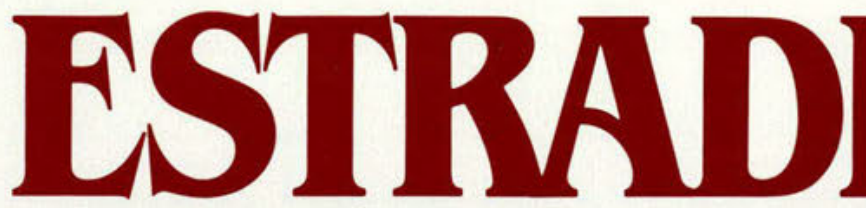 estradiol transdermal syster}

\section{Combines the advantages of transdermal delivery...}

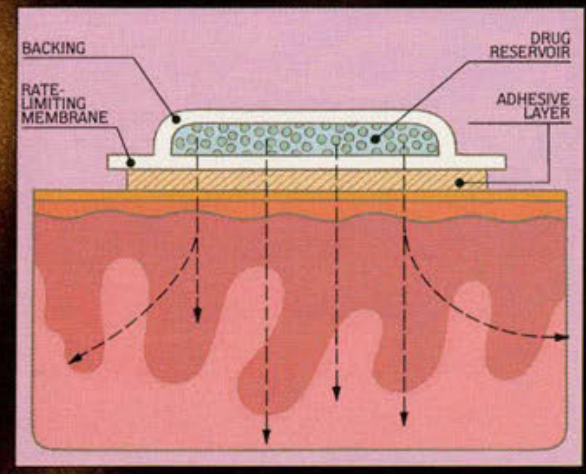

A specially designed transdermal delivery system

Providing the natural ovarian estrog (17-beta estradiol) directly to the bloodstream and target organs; with no first-pass liver metabolism, perm. use of extremely low dosage

Effectively controls estrogen deficiency symptoms

Steady-state relief of hot flashes, oth menopausal symptoms associated w hot flashes, and vaginal discomfort

Well tolerated

Very low incidence of systemic side effects...few local problems of consequence

Easy to use; transdermal delivery preferred 3:1 by patients over tablets $^{1}$

Usual starting dose: a 0.05-mg syste changed twice weekly 


\section{ESTRADERM \\ estradiol transdermal system}

Continuous delivery for twice-weekly application.

BRIEF SUMMARY OF PRESCRIBING INFORMATION.

PLEASE SEE FULLL PRESCRIBING INFORMATION.

\section{ESTROGENS HAVE BEEN REPORTED TO INCREASE THE RISK OF ENDOMETRIAL CARCINOMA.}

Three independent case control studies have reported an increased risk of endometrial cancer in postmenopausal women exposed to exogenous estrogens for more than 1 year. This risk was supported by the finding that incidence rates of endometrial cancer have increased sharply since 1969 in eight different areas of the United States with population-based cancer-reporting systems. an increase which may be related to the rapidly expanding use of estrogens during the last decade. The three case control studies reported that the risk of endometrial cancer in estrogen users was about 4.5-13.9 times greater than in nonusers. The risk appears to depend both on duration of treatment and on estrogen dose. In view of these findings, when estrogens are used for the treatment and on estrogen dose. In view of these findings, when estrogens are used for the
treatment of menopausal symptoms, the lowest dose that will control symptoms should be utilized and medication should be discontinued as soon as possible. When prolonged treatment is medically indicated, the patient should be reassessed on at least a semiannual basis to determine the need for continued therapy. Although the evidence must be considered preliminary. one study suggests that cyclic administration of low doses of estrogen may carry less risk than continuous administration; it cyclic administration of low doses of estrogen may car
therefore appears prudent to utilize such a regimen.

Close clinical surveillance of all wornen taking estrogens is important. In all cases of undiagnosed persistent or recurring abnormal vaginal bleeding, adequate diagnostic measures should be undertaken to rule out malignancy.

There is no evidence at present that "natural" estrogens are more or less hazardous than "synthetic" estrogens at equiestrogenic doses.

\section{ESTROGENS SHOULD NOT BE USED DURING PREGNANCY.}

The use of female sex hormones, both estrogens and progestogens, during early pregnancy may seriously damage the offspring. It has been shown that women who had been exposed in utero to diethylstilbestrol, a nonsteroidal estrogen, have an increased risk of developing in later life a form of vaginal or cervical cancer that is ordinarily extremely rare. This risk has been estimated as not greater than four per 1000 exposures. Furthermore. a high percentage of such exposed women (30-90\%)
have been found to have vaginal adenosis, epithelial changes of the vagina and cervix. Although these changes are histologically benign, it is not known whether they are precursors of malignancy. Although similar data on the use of other estrogens are not available, it cannot be presumed they would not induce similar changes.

Several reports suggest an association between intrauterine exposure to female sex hormones and congenital anomalies, including congenital heart defects and limb-reduction defects. One cas control study estimated a 4.7-fold increased risk of limb-reduction defects in infants who had been exposed in utero to sex hormones (oral contraceptives, hormone withdrawal tests for pregnancy. of attempted treatment for threatened abortion). Some of these exposures were very short and involved only a few days of treatment. The data suggest that the risk of limb-reduction defects in xposed fetuses is somewhat less than one per 1000 .

In the past. female sex hormones have been used during pregnancy in an attempt to treat threatened or habitual abortion. There is considerable evidence that estrogens are ineffective for these indications, and there is no evidence from well-controlled studies that progestogens are

If Estraderm is used

If Estraderm is used during pregnancy. or if the patient becomes pregnant while taking this drug. she should be apprised of the potential risks to the fetus and of the advisability of continuation of the pregnancy.

\section{INDICATIONS AND USAGE}

Estraderm is indicated for the treatment of the following: moderate-to-severe vasomotor symptom associated with menopause: female hypogonadism; female castration; primary ovarian failure; and atrophic conditions caused by deficient endogenous estrogen production, such as atrophic vaginitis and kraurosis vulvae.

Studies of the addition of a progestin for seven or more days of a cycle of estrogen administration have reported a lowered incidence of endometrial hyperplasia. Morphological and biochemical studies of endometrium suggest that 12 to 13 days of progestin are needed to provide maximal maturation of the endometrium and to eliminate any hyperplastic changes. Whether this will provide protection from endometrial carcinoma has not been clearly established. There are possible additional risks that may be associated with the inclusion of progestin in estrogen replacement regimens. The potential risks include adverse effects on carbohydrate and lipid metabolism. The choice of progestin and dosage may be mportant in minimizing these adverse effects.

\section{CONTRAINDICATIONS}

with any of the following conditions:

1. known or suspected cancer of the breast:

2. known or suspected estrogen-dependent neoplasia;

3. known or suspected pregnancy (see Boxed Warning):

4. undiagnosed abnormal genital bleeding:

5. active thrombophlebitis or thromboembolic disorders:

6. history of thrombophlebitis, thrombosis, or thromboembolic disorders associated with previous estrogen use.

\section{WARNINGS}

1. Induction of Malignant Neoplasms. Long-term continuous administration of natural and synthetic estrogens in certain animal species increases the frequency of carcinomas of the breast. cervix. vagina. and (See Boxed Warning)

At the present time

At the present time, there is no satisfactory evidence that estrogens given to postmenopausal women increase the risk of breast cancer, although a recent long-term follow-up of a single physician's practice has raised this possibility. Because of the animal data, there is a need for caution in prescribing estrogens for abnormal mammograms.

2. Gallbladder Disease. A recent study has reported a two- to threefold increase in the risk of surgically confirmed galibladder disease in postmenopausal women receiving oral estrogens, similar to the twofold increase previously noted in users of oral contraceptives.

3. Effects Similar to Those Caused by Estrogen-Progestogen Oral Contraceptives. There are several serious adverse efrects of oral contraceptives and other high-dose oral estrogen treatments, most of therapy. This may reflect the comparatively low doses of estrogen used in postmenopausal women.

a. Thromboembolic Disease. It is now well established that users of oral contraceptives have an
thents a. Thromboembolic Disease. It is now well established that users of oral contraceptives have an
increased risk of various thromboembolic and thrombotic vascular diseases, such as thrombophlebitis, pulmonary embolism, stroke, and myocardial infarction. Cases of retinal thrombosis, mesenteric throm. bosis, and optic neuritis have been reported in oral contraceptive users. There is evidence that the risk of several of these adverse reactions is related to the dose of the drug. An increased risk of postsurgery thromboembolic complications has also been reported in users of oral contraceptives. If feasible. estrogen thromboembolism, or during periods of prolonged immobilization.
While an increased rate of thromboembolic and thrombotic disease in postmenopausal users of estrogens has not been found, this does not rule out the possibility that such an increase may be present or that subgroups of women who have underlying risk factors or who are receiving relatively large doses of estrogens may have increased risk. Therefore, estrogens should not be used in persons with active thrombophlebitis or thromboembolic disorders, and they should not be used in persons with a history of such disorders in association with estrogen use. They should be used with caution in patients with cerebra vascular or coronary artery disease and only for those in whom estrogens are clearly needed.

toses of estrogen ( $5 \mathrm{mg}$ coniugated estrogens per day). comparable to those used to treat cancer of the prostate and breast, have been shown in a large prospective clinical trial in men to increase the risk of nonfatal myocardial infarction, pulmonary embolism, and thrombophlebitis. When estrogen doses of this size are used. any of the thromboembolic and thrombotic adverse effects associated with oral contraceptive use should be considered a clear risk.

b. Hepatic Adenoma. Benign hepatic adenomas have been associated with the use of oral contraceptives. Although benign and rare, these tumors may rupture and cause death from intra-abdominal hemorrhage. Such lesions have not yet been reported in association with other estrogen or progestogen preparations, but they should be considered if abdominal pain and tenderness, abdominal mass, or hypovolemic shock occurs in patients receiving estrogen. Hepatocellular carcinoma has also been reported in women taking estrogen-containing oral contraceptives. The causal relationship of this malignancy to these drugs is not known.

c. Elevated Blood Pressure. Women using oral contraceptives sometimes experience increased blood this mare estrogen use, especially if high dosens in the menopause and blood pressure should be monitored with shown to increase renin substrate. In contrast to these oral estrogens, transdermally administered estradiol does not affect renin substrate.

d. Glucose Tolerance, A worsening of glucose tolerance has been observed in a significant percentage of patients on estrogen-containing oral contraceptives. For this reason, diabetic patients should be carefully while receiving estrogen.

4. Hypercalcemia. Administration of high doses of estrogens may lead to severe hypercalcemia in patients with breast cancer and bone metastases. If hypercalcemia occurs, use of the drug should be PRECAUTIONS

\section{Genera}

1. A complete medical and family history should be taken before initiation of any estrogen therapy. The pretreatment and periodic physical examinations should include special reference to blood pressure. breasts, abdomen, and pelvic organs, as well as a cervical Papanicolaou test. As a general rule, estrogen should not be prescribed for longer than one year without another physical examination being performed 2. Because estrogens may cause some degree of fluid retention, careful observation is required when
conditions that might be influenced by this factor are present (e.g. asthma, epilepsy, migraine, and cardiac conditions that might be

3. Certain patients may develop undesirable manifestations of excessive estrogenic stimulation, such as uterine bleeding, mastodynia, etc.

4. Prolonged administration of unopposed estrogen therapy has been reported to increase the risk of endometrial hyperplasia in some patients. Estrogens should be used with caution in patients who have or have had endometriosis.

5. Oral contraceptives appear to be associated with an increased incidence of mental depression. Although it is not clear whether this is due to the estrogenic or progestogenic component of the contraceptive. patients with a history of depression should be carefully observec.

6. Preexisting uterine leiomyomata may increase in size during prolonged estrogen use. If this occurs, estrogen therapy should be discontinued while the cause is investigated

7. In patients with a history of jaundice during pregnancy. there is an increased risk that jaundice will recur with the use of estrogen-containing oral contraceptives. If jaundice develops in any patient receiving ogen, the medication should be discontinued while the cause is investigated.

8. Estrogens may be poorly metabolized in patients with impaired liver function and should be

a. Because with caution in such patients.

9. Because the prolonged use of estrogens influences the metabolism of calcium and phosphorus, estrogens should be used with caution in patients with metabolic bone diseases associated with hypercal

Drug/Laboratory Test Interactions

The results of certain endocrine and liver function tests may be affected by estrogen-containing oral contraceptives. The following changes have been observed with large doses of oral estrogen:

1. increased sulfobromophthalein retention:

increased prothrombin time: increased factors VII, VIII. IX. and X: decreased antithrombin 3 ; increased norepinephrine-induced platelet aggregability:

3. increased thyroxine-binding globulin (TBG), leading to increased circulating total thyroid hormone $\left(\mathrm{T}_{4}\right)$ as measured by column or radioimmunoassay; free $\mathrm{T}_{3}$ resin uptake is decreased. reflecting the reduced response to the metyrapone test:

5. reduced serum folate concentration:

6. increased serum triglyceride and phospholipid concentration, and decreased pregnanediol excretion. The pathologist should be informed that the patient is receiving estrogen therapy when relevant pecimens are submitted.

\section{Carcinogenesis, Mutagenesis, Impairment of Fertility}

Long-term continuous administration of natural and synthetic estrogens in certain animal species

\section{Pregnancy Category $\mathbf{X}$}

Boxed Warning.

Estrogens should not be used during pregnancy.

\section{Nursing Mothers}

As a general principle, the administration of any drug to nursing mothers should be done only when clearly necessary since many drugs are excreted in human milk

\section{ADVERSE REACTIONS}

See WARNINGS and Boxed Warning regarding potential adverse effects on the fetus, induction of those of oral contraceptives, including thromboembolism.

The most commonly reported adverse reaction to Estraderm in clinical trials was redness and irritation at the application site. This occurred in about $17 \%$ of the women treated and caused approximately $2 \%$ to discontinue therapy.

The following additional adverse reactions have been reported with estrogenic therapy, including oral contraceptives:

Genitourinary Systern; Breakthrough bleeding. spotting. change in menstrual flow: increase in size of terine fibromyomata; change in cervical erosion and amount of cervical secretion.

Endocrine: Breast tenderness, breast enlargement.

( bloating: cholestatic jaundice have been ob-

served with oral estrogen therapy.

Eyes: Steepening of corneal curvature; intolerance to contact lenses.

Central Nervous System: Headache, migraine, dizziness.

Do not store above $86^{\circ} \mathrm{F}\left(30^{\circ} \mathrm{C}\right)$.

Do not store unpouched. Apply immediately upon removal from the protective pouch.

CAUTION: Federal law prohibits dispensing without prescription.

\section{I B A}

CIBA Pharmaceutical Company. Division of CIBA-GEIGY Corporation 